\title{
Targeted high mean arterial pressure aggravates cerebral hemodynamics after extracorporeal resuscitation in swine
}

Yael Levy ${ }^{1,2}$, Alice Hutin ${ }^{1,2,3}$, Fanny Lidouren ${ }^{1,2}$, Nicolas Polge ${ }^{1,2}$, Rocio Fernandez ${ }^{1,2,4}$, Matthias Kohlhauer ${ }^{1,2}$, Pierre-Louis Leger ${ }^{2,5}$, Guillaume Debaty ${ }^{6}$, Keith Lurie ${ }^{7}$, Lionel Lamhaut ${ }^{3}$, Bijan Ghaleh ${ }^{1,2}$ and Renaud Tissier ${ }^{1,2^{*}}$ (D)

\begin{abstract}
Background: Extracorporeal cardiopulmonary resuscitation (E-CPR) is used for the treatment of refractory cardiac arrest. However, the optimal target to reach for mean arterial pressure (MAP) remains to be determined. We hypothesized that MAP levels critically modify cerebral hemodynamics during E-CPR and tested two distinct targets (65-75 vs $80-90 \mathrm{mmHg}$ ) in a porcine model.
\end{abstract}

Methods: Pigs were submitted to 15 min of untreated ventricular fibrillation followed by 30 min of E-CPR. Defibrillations were then delivered until return of spontaneous circulation (ROSC). Extracorporeal circulation was initially set to an average flow of $40 \mathrm{ml} / \mathrm{kg} / \mathrm{min}$. The dose of epinephrine was set to reach a standard or a high MAP target level (65-75 vs 80-90 mmHg, respectively). Animals were followed during 120-min after ROSC.

Results: Six animals were included in both groups. During E-CPR, high MAP improved carotid blood flow as compared to standard MAP. After ROSC, this was conversely decreased in high versus standard MAP, while intra-cranial pressure was superior. The pressure reactivity index (PRx), which is the correlation coefficient between arterial blood pressure and intracranial pressure, also demonstrated inverted patterns of alteration according to MAP levels during E-CPR and after ROSC. In standard-MAP, PRx was transiently positive during E-CPR before returning to negative values after ROSC, demonstrating a reversible alteration of cerebral autoregulation during E-CPR. In high-MAP, PRx was negative during E-CPR but became sustainably positive after ROSC, demonstrating a prolonged alteration in cerebral autoregulation after ROSC. It was associated with a significant decrease in cerebral oxygen consumption in high-versus standard-MAP after ROSC.

Conclusions: During early E-CPR, MAP target above $80 \mathrm{mmHg}$ is associated with higher carotid blood flow and improved cerebral autoregulation. This pattern is inverted after ROSC with a better hemodynamic status with standard versus high-MAP.

Keywords: Cardiac arrest, Resuscitation, Extracorporeal circulation, Epinephrine, Blood pressure

${ }^{*}$ Correspondence: renaud.tissier@vet-alfort.fr

${ }^{1}$ INSERM, IMRB, Univ Paris Est Créteil, 94010 Créteil, France

Full list of author information is available at the end of the article

\section{Introduction}

Out-of-hospital cardiac arrest is the leading cause of mortality in western countries, with an extremely low survival and poor neurological outcome [1]. In order to improve the resuscitation rate, extracorporeal cardiopulmonary resuscitation (E-CPR) is currently suggested, when conventional cardiopulmonary resuscitation 
(CPR) fails to achieve return of spontaneous circulation (ROSC). It allows restoring organ perfusion whilst the underlying etiology is identified and properly treated $[2,3]$. In the clinical arena, E-CPR has been shown to improve survival to hospital discharge and functional status when implemented early, as compared with standard prolonged resuscitation [4, 5]. Nevertheless, the ideal hemodynamic management remains unclear during E-CPR, e.g., regarding optimal mean arterial pressure (MAP) target.

After conventional CPR, several retrospective studies have reported a correlation between favorable outcome and higher MAP target after ROSC [6-8]. Cerebral blood flow and cerebral autoregulation are indeed known to be impaired during the first $72 \mathrm{~h}$ following cardiac arrest in resuscitated patients [9-11]. International guidelines recommend a MAP $>65 \mathrm{mmHg}$ in all resuscitated patients $[12,13]$. However, there is no few specific data regarding cerebral hemodynamics at different MAP levels during E-CPR to our knowledge, despite its relevance as a determinant of cerebral outcome. In swine, no difference on systemic hemodynamics and microcirculation parameters was observed with a MAP set at $65-70$ versus 80-85 mmHg but cerebral hemodynamics and autoregulation were not evaluated [14]. A specific investigation of these parameters is of importance since brain circulation is peculiar with a proper autoregulation and cerebral dramatic metabolic changes after cardiac arrest.

Accordingly, the goal of the present study was to compare the effects of two different MAP targets on cerebral hemodynamics and metabolism during E-CPR $(70 \pm 5$ vs. $85 \pm 5 \mathrm{mmHg}$ ). We used a swine model of cardiac arrest with a prolonged no-flow period of $15 \mathrm{~min}$, in order to mimic a severe hypoxic-ischemic injury that could be observed after refractory cardiac arrest in patients submitted to E-CPR. We started E-CPR directly after the end of the no-flow period to assess its proper effect without other confounding factors secondary to conventional CPR or chest compression.

\section{Methods}

All experiments were reviewed and approved by the ethical committee ComEth Anses-EnvA-UPEC (Committee No. 16, Project \#23076-2019112616472793). All procedures were conducted in accordance with the European Community Standards on the Care and Use of Laboratory Animals.

\section{Animal preparation}

Twelve female swine (27-35 kg) were anesthetized with a mixture of zolazepam and tiletamine $(10 \mathrm{mg} / \mathrm{kg}$, i.m.) followed by propofol ( $1 \mathrm{mg} / \mathrm{kg}$ i.v.). After endotracheal intubation, animals were submitted to conventional mechanical ventilation (tidal volume $=9 \mathrm{ml} / \mathrm{kg}$; $\mathrm{FiO} 2=30 \%$; respiratory rate $=20$ breaths $/ \mathrm{min}$; positive end-expiratory pressure $=5 \mathrm{cmH}_{2} \mathrm{O}$ ). Ventilation parameters were modified when needed to maintain normocapnia et normoxia. Anesthesia was maintained during the instrumentation phase by a continuous administration of propofol $(10 \mathrm{mg} / \mathrm{kg} / \mathrm{h})$. Animals also received methadone $(0.3 \mathrm{mg} / \mathrm{kg}$ i.m. $)$ and rocuronium $(1 \mathrm{mg} / \mathrm{kg}$ i.v. $)$ for analgesia and muscular paralysis, respectively.

Two catheters ( $9 \mathrm{Fr}$ ) were introduced by the Seldinger technique through the right femoral vein and artery for the continuous monitoring of right atrial and systemic arterial blood pressure, respectively. The same technique was used to insert a catheter into the jugular vein for the evaluation of jugular venous oxygen saturation (SjvO2). A $3 \mathrm{~mm}$ blood flow probe (PS-Series Probes ${ }^{\circledR}$, Transonic, NY, USA) was placed around the internal carotid artery to monitor carotid blood flow (CBF). A pressure gauge (Millar ${ }^{\circledR}$, SPR-524, Houston, TX, USA) was inserted into the cerebral cortex after craniotomy to monitor intracranial pressure (ICP). Cerebral oxygen saturation was continuously monitored by near infrared spectroscopy (NIRS; INVOS ${ }^{\mathrm{TM}} 5100 \mathrm{C}$ Cerebral/Somatic Oximeter, Medtronic $\left.{ }^{\circledR}\right)$. Two guidewires were also placed into the left femoral artery and vein for the further insertion of cannulas for extracorporeal membrane oxygenation (ECMO) after induction of cardiac arrest. Unfractionated heparin (100 UI/kg i.v. bolus) was administered immediately after instrumentation. In order to compensate fluid loss, an i.v. infusion of fluid (Ringer lactate, $10 \mathrm{ml} / \mathrm{kg}$ ) was performed during instrumentation.

\section{Cardiac arrest and E-CPR protocol}

After a period of stabilization, ventricular fibrillation (VF) was induced by a pacemaker catheter introduced into the right ventricle through the venous femoral sheath $(\mathrm{A} / \mathrm{C} 10 \mathrm{~V})$. VF was left untreated during $15 \mathrm{~min}$ of no-flow, with no mechanical ventilation. During this period, two 21 and $15 \mathrm{Fr}$ cannulas were mounted around the guidewires previously inserted into femoral vein and artery, respectively (HLS Cannulaes ${ }^{\circledR}$, Maquet, Rastatt, Germany). At the end of the $15 \mathrm{~min}$ of untreated VF, E-CPR was started with a pump flow of $40 \mathrm{ml} / \mathrm{kg} / \mathrm{min}$. The extracorporeal life support circuit included a console, a centrifugal pump (Deltastream ${ }^{\circledR}$ DP3 Pump Heads (Medos Medizintechnik AG, Stolberg,Germany), a membrane oxygenator (PLS-i Oxygenator $^{\circledR}$, Maquet, Rastatt, Germany) and a tubing set (PLS Set ${ }^{\circledR}$, Maquet, Rastatt, Germany). The membrane oxygenator was connected to a mechanical gas blender system (Sechrist Model 20,090 ${ }^{\circledR}$, Sechrist, Anaheim, Calif). The gas flow was adjusted to target a $\mathrm{CO}_{2}$ blood partial pressure between 35 and $45 \mathrm{mmHg}$. After 
30 min of E-CPR, defibrillation attempts were started $(150 \mathrm{~J})$. Mechanical ventilation was resumed immediately after ROSC with the initial parameters. Fluid administration was standardized in all animals with an administration of 15 and $30 \mathrm{ml} / \mathrm{kg}$ of Ringer Lactate immediately after E-CPR initiation and over the two hours following ROSC, respectively. All animals were followed during two hours after ROSC. Then, they were euthanized.

\section{Experimental groups}

As illustrated by Fig. 1a, animals were randomly divided in two experimental groups submitted to different MAP targets after cardiac arrest, i.e., either 65-75 (standard

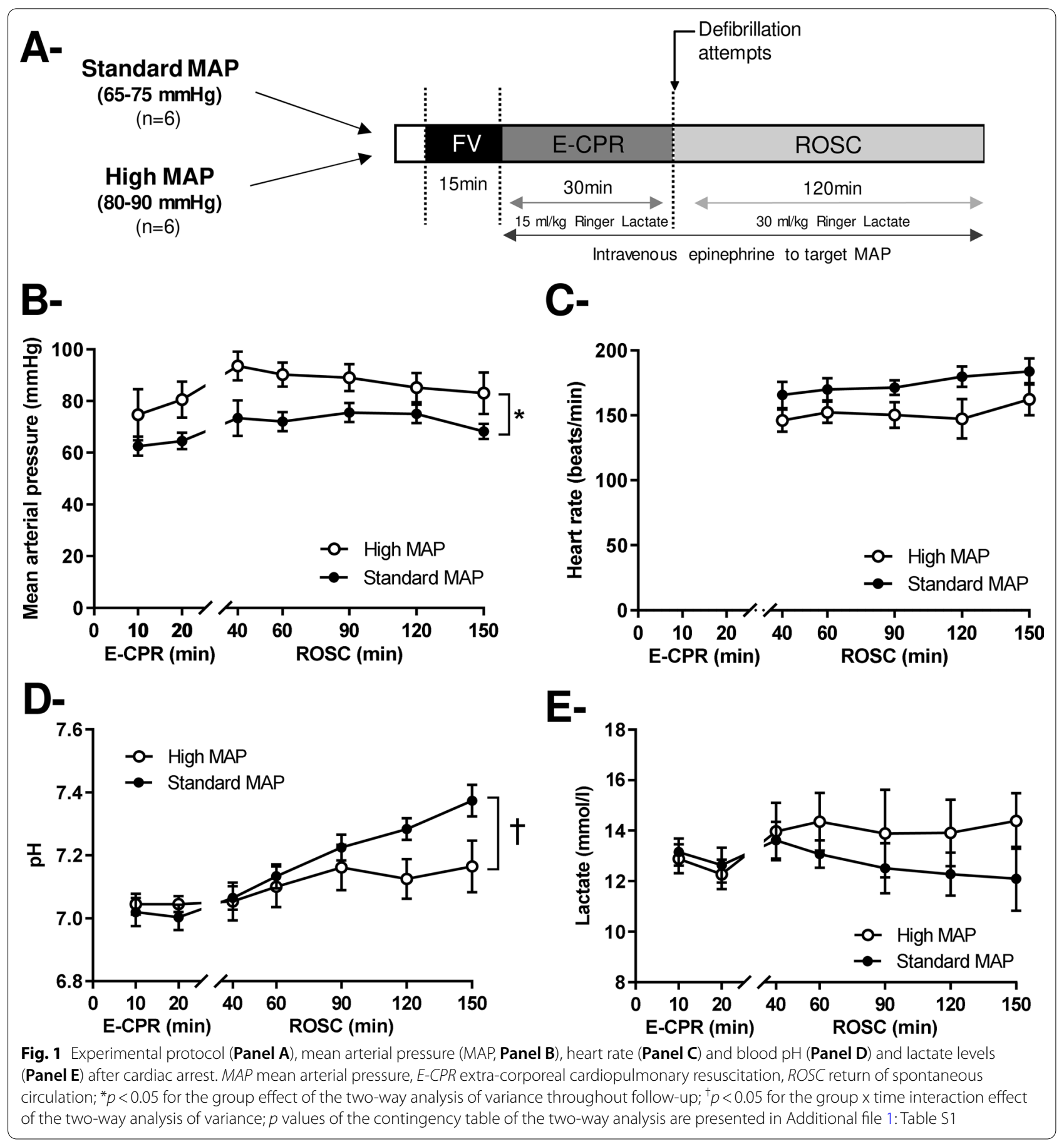


MAP) or $80-90 \mathrm{mmHg}$ (high MAP). This target was achieved by epinephrine administration at adjusted regimen of administration before and after ROSC. We used epinephrine as it is usually recommended during CPR (before ROSC). After cardiac arrest, we targeted a temperature of $37.0 \pm 0.5^{\circ} \mathrm{C}$ using thermal pads and infra-red light.

\section{Investigated parameters}

Heart rate (HR), systolic and diastolic blood pressure, MAP and right atrial pressure were continuously monitored and recorded. CBF, ICP and cerebral NIRS were also monitored and recorded throughout the protocol. Cerebral perfusion pressure was calculated as the difference between MAP and ICP. We also calculated the pressure reactivity index (PRx) as the correlation coefficient between ICP and MAP. It was calculated at baseline and $20,60,90,120$ and 150 min after E-CPR institution during 40 consecutive 6-secondes averages of ICP and MAP, as previously reported [15]. A positive PRx value reflects a nonreactive vascular bed whereas a negative or zero value reflects a normally reactive vascular bed $[15,16]$.

Arterial and venous blood $\mathrm{pH}$, gases $\left(\mathrm{O}_{2}\right.$ and $\mathrm{CO}_{2}$ partial pressure $\left[\mathrm{pO}_{2}\right.$ and $\mathrm{PCO}_{2}$, respectively]), lactates and hemoglobin levels were measured at baseline, during E-CPR ( $t=10$ and $20 \mathrm{~min})$ and after $\operatorname{ROSC}(t=40,60$, $90,120$ and $150 \mathrm{~min})$. The oxygen consumption of the brain hemisphere was approximated using the following equation: $\mathrm{VO}_{2}(\mathrm{ml} \mathrm{O} / \mathrm{min} / \mathrm{kg})=\left(\mathrm{CaO}_{2}-\mathrm{CvO}_{2}\right) \times \mathrm{CBF}$, where $\mathrm{CaO}_{2}$ is arterial oxygen content, $\mathrm{CvO}_{2}$ is internal jugular venous oxygen content, and $\mathrm{CBF}$ is carotid blood flow. $\mathrm{CvO}_{2}$ was calculated as follows: $\mathrm{CvO}_{2}$ $(\mathrm{ml} / \mathrm{l})=\left[\left(1.34 \times \mathrm{Hb}(\mathrm{g} / \mathrm{dl}) \times \mathrm{SvjO}_{2}\right)+\left(0.0031 \times \mathrm{PvjO}_{2}\right)\right] \times$ 10 , where $\mathrm{SvjO}_{2}$ is internal jugular venous oxygen saturation and $\mathrm{PvjO} 2$ is internal jugular venous oxygen tension.

Blood levels of creatinin, alanine aminotransferases (ALT), troponin I and PS100 were also evaluated at baseline and $150 \mathrm{~min}$ after cardiac arrest, as well as interleukin- $1 \alpha(\mathrm{IL}-1 \alpha)$ and interleukin- $1 \beta$ (IL-1 $\beta$; ELISA kits, R\&D Systems, Minneapolis, MN).

\section{Statistical analysis}

Data were expressed as mean \pm SEM. Data normal distribution was verified by a Shapiro-Wilk normality test. At baseline, values were compared among groups using a Student $t$-test. After cardiac arrest, all parameters with repeated measures were compared among groups using a two-way analysis of variance for repeated measures (timing $=10,20,40,60,90,120$ and 150 after the onset of $\mathrm{E}-\mathrm{CPR})$. The $\mathrm{p}$ values of the corresponding time, group and time $\mathrm{x}$ group interaction effects are shown in Additional file 1: Table S1. We only considered the global effects of the ANOVA and did not perform post-hoc comparisons at each time point, in order to avoid multiple comparisons. The parameters with only one measurement at $150 \mathrm{~min}$ after cardiac arrest were compared among groups with a Student $t$-test. A value of $p<0.05$ was considered statistically significant. All statistical analyses were performed using GraphPad Prism ${ }^{\circledR}$ software (GraphPad ${ }^{\circledR}$ Software, La Jolla, CA, USA).

\section{Results}

Twelve swine were enrolled in the present study, i.e., 6 in both standard- and high-MAP groups. Body weights and temperature were not different among groups $(30 \pm 2$ vs $28 \pm 1 \mathrm{~kg}$ and $37.9 \pm 0.3$ vs $37.5 \pm 0.3{ }^{\circ} \mathrm{C}$, respectively), as well as hemodynamic and biochemical parameters at baseline (Table 1). After $30 \mathrm{~min}$ of E-CPR, all animals achieved ROSC after the first electric shock, except one animals which required a second shock in the standardMAP group.

\section{Systemic hemodynamic and blood gases}

As illustrated by Fig. 1b, MAP was maintained at the expected levels in both standard-MAP $(65-75 \mathrm{mmHg})$ and high-MAP (80-90 $\mathrm{mmHg})$. Heart rate was not significantly different among groups (Fig. 1c; Additional file 1: Table S1). Right atrial pressure was not different in standard-MAP vs high -MAP during E-CPR (e.g., $16 \pm 2$ vs $16 \pm 3 \mathrm{mmHg}$ at $\mathrm{t}=20 \mathrm{~min}$ after cardiac arrest) or after ROSC (11 \pm 1 vs $10 \pm 4 \mathrm{mmHg}$ at $t=150 \mathrm{~min}$ after cardiac arrest). The total amount of epinephrine administered to achieve MAP target during the first $30 \mathrm{~min}$ of E-CPR was lower in standard-MAP vs high -MAP group ( $51 \pm 13$ vs $102 \pm 21 \mu \mathrm{g} / \mathrm{kg}$ over $30 \mathrm{~min}$ ). It was also the case after ROSC, until the end of the protocol ( $23 \pm 9$ vs $145 \pm 92 \mu \mathrm{g} / \mathrm{kg}$ over $120 \mathrm{~min}$ in standard-MAP vs highMAP group).

A strong metabolic acidosis was observed in both groups after cardiac arrest, as evidenced by low blood $\mathrm{pH}$ (Fig. 1d), high blood lactate levels (Fig. 1e) and decreased bicarbonate blood levels (e.g., $16 \pm 1$ and $12 \pm 1 \mathrm{mmol} / \mathrm{L}$ in standard-MAP and $17 \pm 1$ and $11 \pm 1 \mathrm{mmol} / \mathrm{L}$ in highMAP at $t=10$ and $150 \mathrm{~min}$ after cardiac arrest). After ROSC, blood $\mathrm{pH}$ was significantly higher in standardversus high-MAP. Lactate blood levels tended to decrease in standard- versus high-MAP but this did not reach statistical significance. $\mathrm{PaO}_{2}$ and $\mathrm{PaCO}_{2}$ did not significantly differ between groups throughout the protocol (data not shown).

\section{Cerebral hemodynamic}

During early E-CPR, CBF was greater in high vs standard-MAP (i.e., before ROSC; Fig. 2a). After ROSC, CBF was conversely lower in high- versus standard-MAP $(p<0.05$ for time $\mathrm{x}$ group interaction, Additional file 1: 
Table 1 Baseline values of investigated parameters

\begin{tabular}{|c|c|c|c|}
\hline Baseline parameters & Standard-MAP N $=6$ & High-MAP N $=6$ & $p$ \\
\hline \multicolumn{4}{|l|}{ Systemic hemodynamic } \\
\hline Heart rate (bpm) & $78 \pm 5$ & $82 \pm 9$ & 0.55 \\
\hline MAP (mmHg) & $95 \pm 16$ & $98 \pm 14$ & 0.54 \\
\hline \multicolumn{4}{|l|}{ Cerebral hemodynamic } \\
\hline Intracranial pressure $\left(\mathrm{cm} \mathrm{H}_{2} \mathrm{O}\right)$ & $10 \pm 1$ & $10 \pm 1$ & 0.84 \\
\hline Cerebral perfusion pressure (mmHg) & $84 \pm 7$ & $87 \pm 6$ & 0.73 \\
\hline Carotid blood flow (ml/min) & $156 \pm 27$ & $137 \pm 8$ & 0.52 \\
\hline Cerebral oxygen consumption $\left(\mathrm{ml} \mathrm{O}_{2} / \mathrm{min} / \mathrm{kg}\right)$ & $45 \pm 3$ & $48 \pm 7$ & 0.63 \\
\hline NIRS cerebral oxygen saturation (\%) & $62 \pm 5$ & $56 \pm 2$ & 0.68 \\
\hline \multicolumn{4}{|l|}{ Blood biochemistry } \\
\hline $\mathrm{pH}$ & $7.46 \pm 0.02$ & $7.42 \pm 0.01$ & 0.21 \\
\hline $\mathrm{PaO}_{2}(\mathrm{mmHg})$ & $158 \pm 3$ & $152 \pm 7$ & 0.68 \\
\hline $\mathrm{PaCO}_{2}(\mathrm{mmHg})$ & $37 \pm 2$ & $40 \pm 2$ & 0.22 \\
\hline $\mathrm{HCO}_{3}^{-}(\mathrm{mmol} / \mathrm{L})$ & $25 \pm 1$ & $25 \pm 2$ & 0.88 \\
\hline Lactate (mmol/L) & $2.5 \pm 0.5$ & $4.4 \pm 1.4$ & 0.34 \\
\hline Hemoglobin (g/dL) & $9.2 \pm 0.4$ & $9.6 \pm 0.4$ & 0.52 \\
\hline
\end{tabular}

Data are presented as Mean \pm SEM

MAP Mean arterial pressure, NIRS near infrared spectroscopy, $\mathrm{PaO}_{2}$ arterial oxygen partial pressure, $\mathrm{PaCO}_{2}$ arterial carbon dioxide partial pressure

Table S1). This was associated with greater amplitude in ICP increase with high- vs standard-MAP after ROSC ( $p<0.05$ for time $\mathrm{x}$ group interaction (Fig. 2b). This led to similar cerebral perfusion pressure among groups at the end of the follow-up Fig. 2c), despite MAP differences.

As illustrated in Fig. 2d, the PRx autoregulation index became transiently positive with standard-MAP just after the onset of E-CPR $(t=20 \mathrm{~min})$, indicating altered cerebral autoregulation. Conversely, PRx was negative during early E-CPR with high-MAP but became sustainably positive after ROSC. If we consider a threshold of +0.2 as a marker of dramatic autoregulation disruption, $5 / 6$ versus $1 / 6$ animals demonstrated values above that threshold in standard vs high-MAP during E-CPR, respectively. At the end of the follow-up $(t=150 \mathrm{~min})$, those numbers were conversely $1 / 6$ and $6 / 6$ in standard vs high-MAP, respectively. This was consistent with the significant decrease in cerebral oxygen consumption in high- versus standard-MAP after ROSC (Fig. 2e). In the same line, cerebral oxygen saturation assessed by NIRS tended to be improved after ROSC with standard versus high-MAP group (Fig. 2f), even if statistical significance was not achieved.

\section{Markers of multi-organ failure and inflammation}

As illustrated in Fig. 3a-d, blood levels of ALT, creatinine, troponin and PS100 similarly increased in both groups after cardiac arrest, demonstrating liver, renal, cardiac and neurological injuries, respectively. The blood levels of IL- $1 \alpha$ and IL-1 $\beta$ also similarly increased after cardiac arrest in both groups (Fig. 3e-f).

\section{Discussion}

In our experimental model of E-CPR, high-MAP transiently improved cerebral hemodynamics after cardiac arrest, as compared to standard-MAP. It was evidenced by higher CBF and lower PRx during early E-CPR. However, this beneficial effect was lost after ROSC and high MAP became deleterious with a dramatic deterioration of cerebral hemodynamics, including lower CBF, positive PRx, increased ICP and deteriorated cerebral oxygen consumption and saturation vs standard-MAP. HighMAP also worsened metabolic acidosis as compared to standard-MAP, despite similar blood levels of biomarkers of end-organ of dysfunction at the end of the follow-up.

In the literature, it is well admitted that cerebral perfusion and autoregulation are strongly impaired and associated with unfavorable neurological outcome after cardiac arrest $[9,11,17]$. Cerebral blood flow is then often believed to directly depends upon perfusion pressure, supporting the putative interest of high-MAP targets. However, MAP support requires the administration of catecholamines exerting their own effects on microvascular beds and cerebral blood flow. It is particularly true with epinephrine which was shown to decrease cerebral microvascular blood flow and increase the severity of cerebral ischemia in swine submitted to cardiac arrest and conventional CPR [18]. This could explain why highMAP was beneficial during the very early phase of E-CPR 


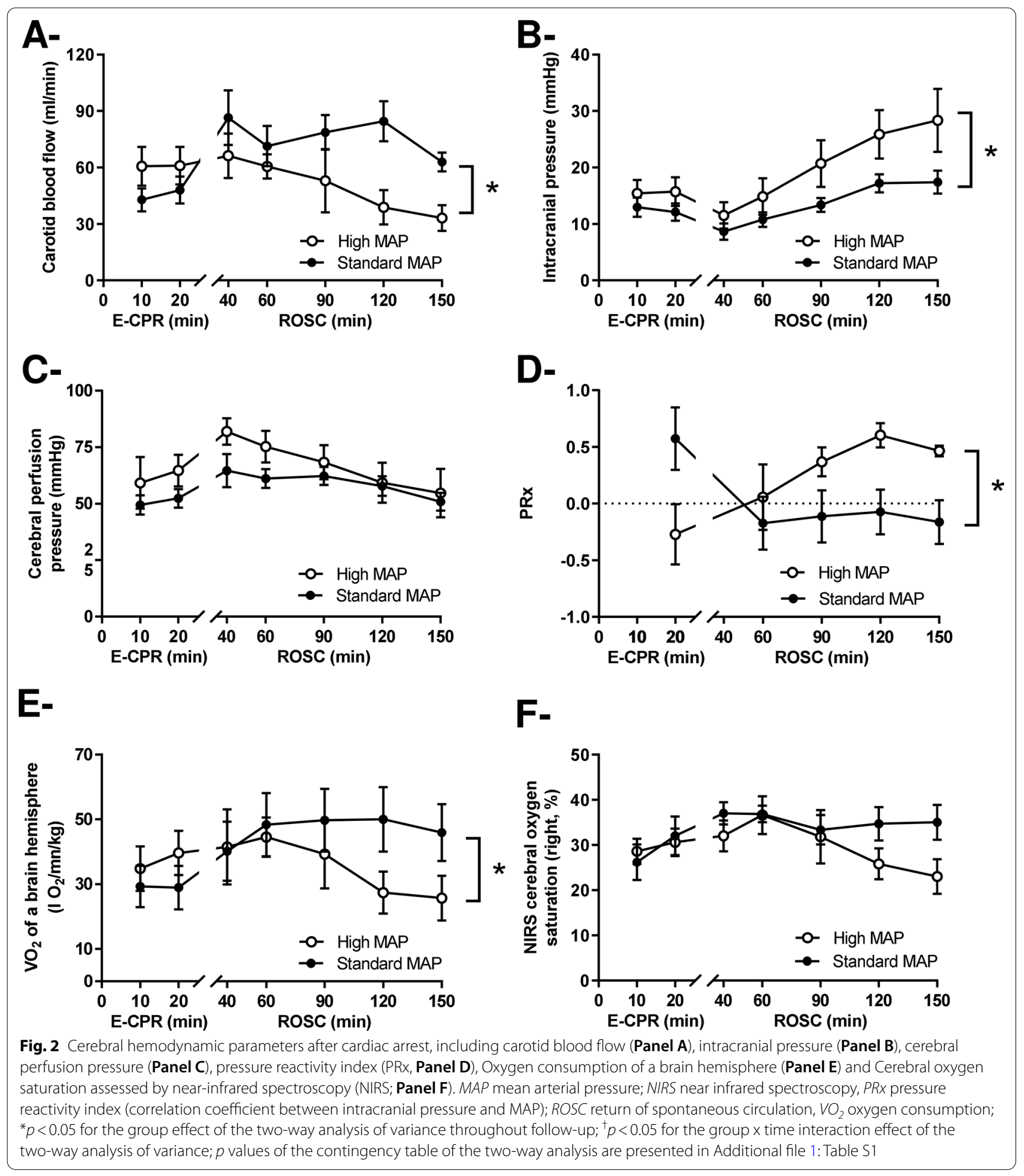

but became clearly detrimental after ROSC in our study. The decrease in cerebral oxygen consumption and CBF could then be related to altered microcirculation and ICP increase, with increased cerebral vascular resistance and cerebral swelling.

In the present study, PRx analyses also demonstrate altered cerebral autoregulation with high-MAP after 


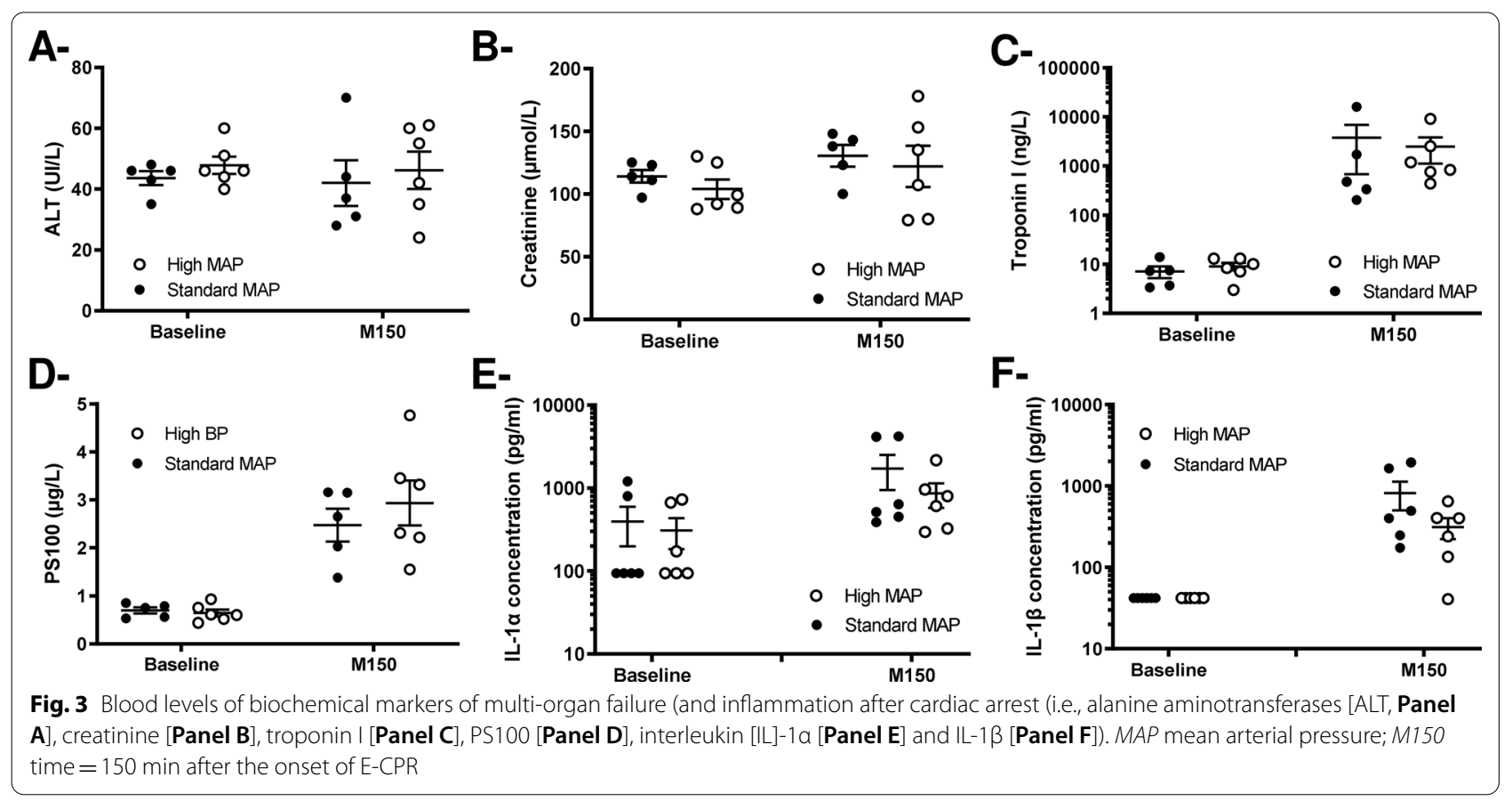

ROSC, with a positive correlation between ICP and MAP after ROSC. Interestingly, PRx values above +0.2 were shown to predict poor neurological outcome with a strong specificity and sensitivity in patients after conventional CPR [19]. When compared to standard-MAP, the high-MAP strategy was therefore clearly deleterious after ROSC, although transiently improving cerebral hemodynamic during the early phase preceding ROSC in our study. One could argue that a double approach should be proposed with a high-MAP target during early E-CPR and then a standard-MAP after ROSC. Unfortunately, such a condition was not included in our randomization plan but clearly deserves further investigation. An investigation with norepinephrine rather than epinephrine would also be relevant after ROSC. Beyond the vasotensive effect of those drugs, the worsening of cerebral hemodynamics with high-MAP could also be indirectly related to altered venous drainage and increased venous congestion and vascular leakage during E-CPR. However, this should not be the major mechanism since ECMO flows, fluid administration and right atrial pressure were similar in standard- and high-MAP groups.

Importantly, the current recommendations for hemodynamic managements of cardiac arrest patients were mostly raised after conventional CPR (i.e., not specifically following E-CPR). A MAP level above $65 \mathrm{mmHg}$ with prevention of hypotension is then proposed [12]. Hypotension is indeed clearly associated with poor neurological outcome $[6,7,20]$, while observational studies showed an improved neurological outcomes with MAP levels set at 70-75 $\mathrm{mmHg}[7,8]$. Importantly, randomized studies did not demonstrate a deleterious effect of higher MAP in those conditions. For instance, the neurological injury evaluated by neural specific enolase blood levels was not different with a MAP of level of 65-75 versus 80-100 mmHg after ROSC [21]. In another study, similar extents of anoxic brain damage were observed with a MAP target of $85-100 \mathrm{mmHg}$, with a mixed venous $\mathrm{O}_{2}$ saturation of $65-75 \%$, when compared to a $65 \mathrm{mmHg}$ MAP strategy [22]. All these studies were obtained after conventional CPR and one would speculate different situations in patients with E-CPR, due to the increased severity of the anoxic brain injury, as suggested by our present findings. This difference should not be so critical for other organ injury and systemic hemodynamics as we did not observe much difference on systemic hemodynamics and other biomarkers, except a significantly higher blood $\mathrm{pH}$ with standard- versus high-MAP after ROSC. This is consistent with the results obtained by Fritz et al. [14] in swine submitted to prolonged cardiac arrest and E-CPR with two different MAP targets $(65-70 \mathrm{mmHg}$ vs $80-85 \mathrm{mmHg}$ ) during $6 \mathrm{~h}$ after ECMO initiation. The latter study did not assess cerebral hemodynamics to our knowledge. Longer follow-up duration would also evidence differences on the longer term.

In summary, our experimental study supports the use of a $65-75 \mathrm{mmHg}$ MAP target in patients under ECMO, at least after ROSC. Specific clinical trials are still 
required but our findings, along with most clinical data in patients resuscitated after convention CPR, indeed demonstrates the lack of benefit, or even the deleterious effect, of high- vs standard-MAP target. We should point that this putative recommendation could only be relevant for MAP target with vasopressors, which does not mean that similar levels of MAP could lead to similar observations in patients weaned from vasotensive agents.

\section{Study limitations}

Our present study presents several limitations. As already discussed, we used epinephrine to achieve the MAP target in both groups since we initially focused on the early E-CPR preceding ROSC, for which epinephrine is likely the most relevant drug. It would have been difficult to interpret the results if using different drugs after ROSC, e.g., dobutamine and norepinephrine. If norepinephrine is usually recommended after ROSC [12], a recent Cochrane review did not find any difference in mortality with six different vasopressors assessed in 3497 patients presenting hypotensive shock [23]. Second, another important limitation is the short follow-up duration of the study, which did not allow the evaluation of the long term neurological outcome. Cerebral imaging or histological analyses would also have been of high interest but they were not conducted in the present study. We can only raise our conclusions on surrogate markers such as the PRx as an autoregulation parameter. Third, we only follow animals during a short period that makes the analysis of biomarkers data difficult. Finally, such experimental studies are typically done using a limited number of animals. Since we only investigated females, it is also uncertain that the results could be generalized to males.

\section{Conclusions}

In an experimental swine model of refractory cardiac arrest treated by E-CPR, a high-MAP target improved hemodynamics during the early phase of E-CPR preceding ROSC, when compared to standard-MAP. Conversely, high-MAP became highly deleterious after resuscitation. This could support a combined strategy", with an initial aggressive MAP control followed by a more conservative strategy after E-CPR.

\section{Supplementary Information}

The online version contains supplementary material available at https://doi. org/10.1186/s13054-021-03783-3.

Additional file 1. Supplemental Table I: P values of the contingency table of the two-way analysis of variance (ANOVA) for repeated measures with group, time effect and group $x$ time interaction effects for the different investigated parameters after cardiac arrest.
Acknowledgements

Not applicable.

\section{Authors' contributions}

$Y L, G D, K L, P L L, B G, L L$ and RT conceived the study, designed the trial, and/or obtained research funding. $Y L, A H, F L, R F, M K, B G$ and RT supervised the conduct of the trial and data collection. YL, AH, FL, NM, RF and RT were caregivers during experiment and managed the data, including quality control. YL and RT provided statistical advice on study design and analyzed the data. YL and RT drafted the manuscript, and all authors contributed substantially to its revision. All authors take responsibility for the paper as a whole. All authors read and approved the final manuscript.

\section{Funding}

The study was supported by Grant COOLIVENT by Agence Nationale pour la Recherche. Yaël Levy was supported by ADEREM.

Availability of data and materials

The datasets used and/or analysed during the current study are available from the corresponding author on reasonable request.

\section{Declarations}

\section{Ethics approval}

All experiments were reviewed and approved by the ethical committee ComEth Anses-EnvA-UPEC (Committee No. 16, Project \#23076-2019112616472793).

\section{Consent for publication}

Not applicable.

\section{Competing interests}

M Kohlhauer and R Tissier are shareholders of a start-up company dedicated to total liquid ventilation (Orixha).

\section{Author details}

${ }^{1}$ INSERM, IMRB, Univ Paris Est Créteil, 94010 Créteil, France. ${ }^{2}$ IMRB, AfterROSC Network, Ecole Nationale Vétérinaire d'Alfort, 7 Avenue du Général de Gaulle, 94700 Maisons-Alfort, France. ${ }^{3}$ Assistance Publique-Hôpitaux de Paris, SAMU de Paris-ICU, Necker University Hospital, Université de Paris, 75015 Paris, France. ${ }^{4}$ Department of Small Animal Medicine and Surgery, Faculty of Veterinary Medicine, Catholic University of Valencia, 46001 Valencia, Spain. ${ }^{5}$ Assistance Publique-Hôpitaux de Paris, Hôpital Trousseau, Université de Paris, Sorbonne Université, 75012 Paris, France. ${ }^{6}$ Department of Emergency Medicine, CNRS, TIMC Laboratory - UMR 5525, Grenoble Alpes University Hospital, University Grenoble Alpes, Grenoble, France. ${ }^{7}$ Hennepin Healthcare Research Institute, Minneapolis, MN, USA

Received: 17 August 2021 Accepted: 6 October 2021

Published online: 14 November 2021

\section{References}

1. Berdowski J, Berg RA, Tijssen JGP, Koster RW. Global incidences of out-ofhospital cardiac arrest and survival rates: systematic review of 67 prospective studies. Resuscitation. 2010;81:1479-87.

2. Chen LM, Nallamothu BK, Spertus JA, Li Y, Chan PS, American Heart Association's get with the guidelines-resuscitation investigators. Association between a hospital's rate of cardiac arrest incidence and cardiac arrest survival. JAMA Intern Med 2013;173:1186-1195.

3. Lamhaut L, Hutin A, Puymirat E, Jouan J, Raphalen J-H, Jouffroy R, Jaffry M, Dagron C, An K, Dumas F, Marijon E, Bougouin W, Tourtier J-P, Baud F, Jouven X, Danchin N, Spaulding C, Carli P. A Pre-Hospital Extracorporeal Cardio Pulmonary Resuscitation (ECPR) strategy for treatment of refractory out hospital cardiac arrest: an observational study and propensity analysis. Resuscitation. 2017;117:109-17.

4. Stub D, Bernard S, Pellegrino V, Smith K, Walker T, Sheldrake J, Hockings L, Shaw J, Duffy SJ, Burrell A, Cameron P, Smit DV, Kaye DM. Refractory 
cardiac arrest treated with mechanical CPR, hypothermia, ECMO and early reperfusion (the CHEER trial). Resuscitation. 2015;86:88-94.

5. Yannopoulos D, Bartos J, Raveendran G, Walser E, Connett J, Murray TA, Collins G, Zhang L, Kalra R, Kosmopoulos M, John R, Shaffer A, Frascone RJ, Wesley K, Conterato M, Biros M, Tolar J, Aufderheide TP. Advanced reperfusion strategies for patients with out-of-hospital cardiac arrest and refractory ventricular fibrillation (ARREST): a phase 2 , single centre, openlabel, randomised controlled trial. Lancet Lond Engl. 2020;396:1807-16.

6. Laurikkala J, Wilkman E, Pettilä V, Kurola J, Reinikainen M, Hoppu S, Ala-Kokko T, Tallgren M, Tiainen M, Vaahersalo J, Varpula T, Skrifvars MB, FINNRESUSCI Study Group. Mean arterial pressure and vasopressor load after out-of-hospital cardiac arrest: associations with one-year neurologic outcome. Resuscitation 2016;105:116-122.

7. Russo JJ, Di Santo P, Simard T, James TE, Hibbert B, Couture E, Marbach J, Osborne C, Ramirez FD, Wells GA, Labinaz M, Le May MR, from the CAPITAL study group. Optimal mean arterial pressure in comatose survivors of out-of-hospital cardiac arrest: an analysis of area below blood pressure thresholds. Resuscitation 2018;128:175-180.

8. Kilgannon JH, Roberts BW, Jones AE, Mittal N, Cohen E, Mitchell J, Chansky ME, Trzeciak S. Arterial blood pressure and neurologic outcome after resuscitation from cardiac arrest*. Crit Care Med. 2014;42:2083-91.

9. Lemiale V, Huet O, Vigué B, Mathonnet A, Spaulding C, Mira J-P, Carli P, Duranteau J, Cariou A. Changes in cerebral blood flow and oxygen extraction during post-resuscitation syndrome. Resuscitation. 2008;76:17-24.

10. Bisschops LLA, Hoedemaekers CWE, Simons KS, van der Hoeven JG. Preserved metabolic coupling and cerebrovascular reactivity during mild hypothermia after cardiac arrest. Crit Care Med. 2010;38:1542-7.

11. Sundgreen C, Larsen FS, Herzog TM, Knudsen GM, Boesgaard S, Aldershvile J. Autoregulation of cerebral blood flow in patients resuscitated from cardiac arrest. Stroke. 2001;32:128-32.

12. Nolan JP, Sandroni C, Böttiger BW, Cariou A, Cronberg T, Friberg H, Genbrugge C, Haywood K, Lilja G, Moulaert VRM, Nikolaou N, Olasveengen TM, Skrifvars MB, Taccone F, Soar J. European resuscitation council and European society of intensive care medicine guidelines 2021: post-resuscitation care. Intensive Care Med. 2021;47:369-421.

13. Panchal AR, Bartos JA, Cabañas JG, Donnino MW, Drennan IR, Hirsch KG, Kudenchuk PJ, Kurz MC, Lavonas EJ, Morley PT, O'Neil BJ, Peberdy MA, Rittenberger JC, Rodriguez AJ, Sawyer KN, Berg KM, Adult Basic and Advanced Life Support Writing Group. Part 3: adult basic and advanced life support: 2020 American Heart Association guidelines for cardiopulmonary resuscitation and emergency cardiovascular care. Circulation 2020;142:S366-S468

14. Fritz C, Kimmoun A, Vanhuyse F, Trifan BF, Orlowski S, Falanga A, Marie V, Groubatch F, Albuisson E, Tran N, Levy B. High versus low blood-pressure target in experimental ischemic prolonged cardiac arrest treated with extra corporeal life support. Shock Augusta Ga. 2017:47:759-64.

15. Czosnyka M, Smielewski P, Kirkpatrick P, Laing RJ, Menon D, Pickard JD Continuous assessment of the cerebral vasomotor reactivity in head injury. Neurosurgery. 1997:41:11-7 (discussion 17-19).

16. Steiner LA, Czosnyka M, Piechnik SK, Smielewski P, Chatfield D, Menon DK, Pickard JD. Continuous monitoring of cerebrovascular pressure reactivity allows determination of optimal cerebral perfusion pressure in patients with traumatic brain injury. Crit Care Med. 2002;30:733-8.

17. Ameloot K, Genbrugge C, Meex I, Jans F, Boer W, Vander Laenen M, Ferdinande B, Mullens W, Dupont M, Dens J, DeDeyne C. An observational near-infrared spectroscopy study on cerebral autoregulation in post-cardiac arrest patients: time to drop "one-size-fits-all" hemodynamic targets? Resuscitation. 2015;90:121-6.

18. Ristagno G, Tang W, Huang L, Fymat A, Chang Y-T, Sun S, Castillo C, Weil $\mathrm{MH}$. Epinephrine reduces cerebral perfusion during cardiopulmonary resuscitation. Crit Care Med. 2009;37:1408-15.

19. Balu R, Rajagopalan S, Baghshomali S, Kirschen M, Amurthur A, Kofke WA, Abella BS. Cerebrovascular pressure reactivity and intracranial pressure are associated with neurologic outcome after hypoxic-ischemic brain injury. Resuscitation. 2021. https://doi.org/10.1016/j.resuscitation.2021.04. 023.

20. Annoni F, Dell'Anna AM, Franchi F, Creteur J, Scolletta S, Vincent J-L, Taccone FS. The impact of diastolic blood pressure values on the neurological outcome of cardiac arrest patients. Resuscitation. 2018;130:167-73.

21. Jakkula P, Pettilä V, Skrifvars MB, Hästbacka J, Loisa P, Tiainen M, Wilkman E, Toppila J, Koskue T, Bendel S, Birkelund T, Laru-Sompa R, Valkonen M, Reinikainen M, COMACARE study group. Targeting low-normal or highnormal mean arterial pressure after cardiac arrest and resuscitation: a randomised pilot trial. Intensive Care Med 2018;44:2091-2101.

22. Ameloot K, De Deyne C, Eertmans W, Ferdinande B, Dupont M, Palmers P-J, Petit T, Nuyens P, Maeremans J, Vundelinckx J, Vanhaverbeke M, Belmans A, Peeters R, Demaerel P, Lemmens R, Dens J, Janssens S. Early goal-directed haemodynamic optimization of cerebral oxygenation in comatose survivors after cardiac arrest: the Neuroprotect post-cardiac arrest trial. Eur Heart J. 2019;40:1804-14.

23. Gamper G, Havel C, Arrich J, Losert H, Pace NL, Müllner M, Herkner H. Vasopressors for hypotensive shock. Cochrane Database Syst Rev. 2016;2:CD003709.

\section{Publisher's Note}

Springer Nature remains neutral with regard to jurisdictional claims in published maps and institutional affiliations.

\footnotetext{
Ready to submit your research? Choose BMC and benefit from:

- fast, convenient online submission

- thorough peer review by experienced researchers in your field

- rapid publication on acceptance

- support for research data, including large and complex data types

- gold Open Access which fosters wider collaboration and increased citations

- maximum visibility for your research: over $100 \mathrm{M}$ website views per year
}

At BMC, research is always in progress.

Learn more biomedcentral.com/submissions 